\title{
Setting Fees in Competing Double Auction Marketplaces: An Equilibrium Analysis
}

\author{
Bing Shi, Enrico H. Gerding, Perukrishnen Vytelingum and Nicholas R. \\ Jennings
}

School of Electronics and Computer Science,
University of Southampton, Southampton, UK
\{bs07r, eg, pv, nrj\}@ecs.soton.ac.uk

\begin{abstract}
In this paper, we analyse competing double auction marketplaces that vie for traders and need to set appropriate fees to make a profit. Specifically, we show how competing marketplaces should set their fees by analysing the equilibrium behaviour of two competing marketplaces. In doing so, we focus on two different types of market fees: registration fees charged to traders when they enter the marketplace, and profit fees charged to traders when they make transactions. In more detail, given the market fees, we first derive equations to calculate the marketplaces' expected profits. Then we analyse the equilibrium charging behaviour of marketplaces in two different cases: where competing marketplaces can only charge the same type of fees and where competing marketplaces can charge different types of fees. This analysis provides insights which can be used to guide the charging behaviour of competing marketplaces. We also analyse whether two marketplaces can co-exist in equilibrium. We find that, when both marketplaces are limited to charging the same type of fees, traders will eventually converge to one marketplace. However, when different types of fees are allowed, traders may converge to different marketplaces (i.e. multiple marketplaces can co-exist).
\end{abstract}

Key words: Competing Marketplaces, Nash Equilibrium, Evolutionary Game Theory, Double Auctions

\section{Introduction}

Financial exchanges, in which securities, futures, stocks and commodities can be traded, are becoming ever more prevalent. Now, many of these adopt the double auction market mechanism which is a particular type of two-sided market with multiple buyers (one side) and multiple sellers (the other side). Specifically, in such a mechanism, traders can submit offers at any time in a specified trading round, and can be matched by the marketplace at a specified time. The advantages of this mechanism are that traders can enter the marketplace at any time and they can trade multiple homogeneous or heterogeneous items in one place without travelling around several marketplaces. In addition, this mechanism provides high allocative efficiency [3]. These benefits have led many electronic marketplaces to also use this format. For example, Google owns Dou- 
bleClick Ad Exchange ${ }^{1}$, which is a real-time double auction marketplace enabling large online ad publishers, on one side, and ad networks and agencies, on the other side, to buy and sell advertising space. However, because of the globalised economy, these marketplaces do not exist in isolation. Thus they compete against each other to attract traders and make profits by charging fees to traders. For example, stock exchanges compete to attract companies to list their stocks in their marketplaces and make profits by charging listing fees to these companies, and Google competes against other ad exchanges, such as Microsoft's AdECN and Yahoo!'s Right Media. However, there exists a conflict between attracting traders and making profits for the competing marketplace, since when the fees are increased, traders will leave the marketplace and eventually cause a decrease of profits for this marketplace. Against this background, in this paper, we analyse the equilibrium behaviour of competing marketplaces in terms of charging fees to traders, which can provide insights to guide how competing marketplaces should set their fees.

In more detail, there are two key issues in the research of competing marketplaces. The first is how traders should choose which marketplace to go to. Then, given the traders' market selection strategies, the second issue is how competing marketplaces should set their fees to maximise profits while at the same time maintaining market share at a good level in order to ensure profits in the long term. Now we have analysed the first issue in our previous work [1], so here we focus on how competing marketplaces set their fees.

Related to our work, a number of theoretical models have been proposed to analyse two-sided competing marketplaces (e.g. $[2,5,6,8]$ ). However, these works do not consider auction mechanisms to match traders and set transaction prices. Instead, they assume that traders only select marketplaces based on the number of other traders in the marketplace. In doing so, they assume that all traders are homogeneous (i.e. have the same preferences), and the marketplace has complete information about the preferences (also called the types) of traders. In real-world auction marketplaces, however, traders are usually heterogeneous and they are likely to have privately known preferences. Moreover, transaction prices are usually set according to the marketplace's pricing policy, which is affected by current demand and supply. Also related to our work is the Market Design Competition (CAT), an annual competition and part of the Trading Agent Competition (TAC) which was introduced to promote research in the area of competing double auctions [4]. However, the work related to CAT is still largely empirical in nature. To tackle these limitations, in our previous work [1], we proposed a novel game-theoretic framework to analyse the competition between double auction marketplaces from a theoretical perspective, which assumes that traders are heterogeneous with different types ${ }^{2}$ and the type of each specific trader is not known to the other traders and marketplaces. Based on this framework, we analysed the traders' Nash equilibrium (NEQ) market

\footnotetext{
1 http://www.doubleclick.com/

2 The types of buyers and sellers represent the buyers' limit prices and the sellers' cost prices respectively. The limit price is the highest price that the buyer is willing to buy the item for, and the cost price is the lowest price that the seller is willing to sell the item for.
} 
selection strategies. Moreover, using evolutionary game theory (EGT) [10], we analysed how traders dynamically change their market selection strategies and determined which strategies traders eventually converge to.

In this paper, we extend this work by analysing how double auction marketplaces set their fees to make profits in a multiple competing marketplaces environment. In this environment, if the marketplace charges higher fees than its opponents, then it may make more profits in the short-term. However, eventually traders will choose to leave this marketplace and choose to migrate to the cheaper marketplace. This will therefore result in a decrease of profits. Thus the competing marketplace should charge appropriate fees to make profit and maintain market share given its opponents' fees. In this paper, we will analyse this pattern by considering the equilibrium charging behaviour between marketplaces. In reality, two types of fees are usually charged to traders. One is the ex-ante fee charged to traders before they make transactions. The other is the ex-post fee charged conditional on traders making a transaction [5]. Specifically, in our analysis, we consider the registration fee charged to traders when they enter the marketplace, and the profit fee charged to traders when they make transactions as a typical example of ex-ante and ex-post fees respectively. Furthermore, we analyse under what conditions several competing double auction marketplaces can co-exist when traders converge to their equilibrium market selection strategies. That is, we are interested in analysing whether competition can be maintained, or whether the marketplaces collapse to a monopoly setting where all traders move to one marketplace. This is important since competition drives efficiency and offers more and better choices to traders. In previous work, we found when competing marketplaces are only allowed to charge the profit fees, traders eventually converge to one marketplace. In [2] which considers the competition of two-sided marketplaces, researchers claim that when two competing marketplaces differentiate themselves from each other, they may co-exist. In this paper, we will analyse the co-existing issue in the context of competing double auction marketplaces.

In particular, the contributions of this paper are as follows. First, we provide a novel approach to estimate marketplaces' expected profits given the equilibrium strategies of the traders and the fees charged by other marketplaces. Second, based on the estimated expected profits of marketplaces, we are the first to analyse the equilibrium charging behaviour of competing double auction marketplaces. Finally, we show that in our framework, when different types of fees are allowed, traders may converge to different marketplaces, i.e. competing marketplaces can co-exist.

The structure of the paper is as follows. In Section 2, we briefly describe the general framework from [1] for analysing competing double auction marketplaces. In Section 3, we provide an approach to estimate marketplaces' expected profits. Then in Section 4, we analyse the equilibrium for two settings: when competing marketplaces charge the same type of fees, and with different type of fees. We also investigate under what under conditions competing marketplaces co-exist. Finally, we conclude this paper in Section 5. 


\section{General Framework}

In this section, we briefly introduce the framework developed in [1]. We start by introducing basic notations of our framework. Then we introduce the marketplaces and their policies. Finally, we describe the market selection strategies in detail and give a general equation for a trader's expected utility.

\subsection{Preliminaries}

We assume that there are a set of buyers, $\mathcal{B}=\{1,2, \ldots B\}$, and a set of sellers, $\mathcal{S}=\{1,2, \ldots S\}$. Each buyer and seller has a type, which is denoted as $\theta^{b}$ and $\theta^{s}$ respectively. We assume that types of all buyers are independently drawn from the same cumulative distribution function $F^{b}$, with support $[\underline{l}, \bar{l}]$, and the types of all sellers are independently drawn from the cumulative distribution function $F^{s}$, with support $[\underline{c}, \bar{c}]$. The distributions $F^{b}$ and $F^{s}$ are assumed to be common knowledge and differentiable. The probability density functions are $f^{b}$ and $f^{s}$ respectively. In our framework, the type of each specific trader is not known to the other traders and marketplaces, and only the type distribution functions are public. In addition, we assume that there is a set of competing marketplaces $\mathcal{M}=\{1,2, \ldots M\}$, that offer places for trade and provide a matching service between the buyers and sellers.

\section{$2.2 \quad$ Marketplaces and Fees}

Since we consider marketplaces to be commercial enterprises that seek to make a profit, we assume they charge fees for their service as match makers. The fee structure of a marketplace $m$ is defined, as $\mathcal{P}_{m}=\left\langle p_{m}^{b}, p_{m}^{s}, q_{m}^{b}, q_{m}^{s}\right\rangle, p_{m}^{b}, p_{m}^{s} \geq 0$ and $q_{m}^{b}, q_{m}^{s} \in[0,1]$, where $p_{m}^{b}, p_{m}^{s}$ are fixed flat fees charged to buyers and sellers respectively (in this paper, as an example, we consider registration fees charged to traders when they enter the marketplace as a typical kind of fixed flat fee), and $q_{m}^{b}, q_{m}^{s}$ are percentage fees charged on profits made by buyers and sellers respectively (in the following, we refer to such fees as profit fees). Then the fees of all competing marketplaces constitute the fee system $\mathcal{P}=\left\langle\mathcal{P}_{1}, \mathcal{P}_{2}, \ldots \mathcal{P}_{M}\right\rangle$. In addition, we use $\mathcal{P}_{-m}$ to represent fees of all marketplaces except for marketplace $m$. Then $\mathcal{P}$ can be rewritten as $\mathcal{P}=\left\langle\mathcal{P}_{m}, \mathcal{P}_{-m}\right\rangle$. Furthermore, the transaction price of a successful transaction in marketplace $m$ is determined by a parameter $k_{m} \in[0,1]$, i.e. a discriminatory $k$-pricing policy, which sets the transaction price of a matched buyer and seller at the point determined by $k_{m}$ in the interval between their offers. The pricing parameters of all marketplaces constitute the pricing system $\mathcal{K}=\left\langle k_{1}, k_{2}, \ldots, k_{M}\right\rangle$.

\subsection{Trader Market Selection}

We assume that traders can only choose a single marketplace at a time (called single-homing), but they can freely migrate to a different marketplace in the next trading round. A trading round proceeds as follows. First, all marketplaces 
publish their fees and pricing parameters. Second, based on the observed fees and pricing parameters, each trader selects a marketplace according to its market selection strategy. Third, traders submit their offers according to their bidding strategies. Finally, after all traders have submitted their offers, the marketplace matches buyers and sellers according to its matching policy and then executes transactions. For simplicity, we assume that only one unit of commodity can be traded by each trader in a giving trading round. Intuitively, we can see that the traders' choice of marketplaces is important since this significantly affects the marketplaces' positions in the competition. Given this, in the following, we present the traders' market selection strategies in more detail.

We consider a mixed market selection strategy, where each marketplace is selected with some probability. A pure strategy can be regarded as a degenerate case of a mixed strategy, where the particular pure strategy is selected with probability 1 and every other strategy with probability 0 . Now, a mixed market selection strategy of buyer $i$ is defined as $\omega_{i}^{b}:[\underline{l}, \bar{l}] \times \mathcal{M} \rightarrow[0,1]$, which means the probability that buyer $i$ with type $\theta^{b}$ chooses the marketplace $m$ is $\omega_{i}^{b}\left(\theta^{b}, m\right)$, where $\sum_{m \in \mathcal{M}} \omega_{i}^{b}\left(\theta^{b}, m\right) \leq 1$. Here, $1-\sum_{m \in \mathcal{M}} \omega_{i}^{b}\left(\theta^{b}, m\right)$ is the probability that buyer $i$ with type $\theta^{b}$ chooses no marketplace. The complete mixed market selection strategy of buyer $i$ with type $\theta^{b}$ is given by:

$$
\delta_{i}^{b}\left(\theta^{b}\right)=\left\langle\omega_{i}^{b}\left(\theta^{b}, 1\right), \omega_{i}^{b}\left(\theta^{b}, 2\right), \ldots \omega_{i}^{b}\left(\theta^{b}, M\right)\right\rangle, \quad \delta_{i}^{b}\left(\theta^{b}\right) \in \Delta,
$$

where $\Delta$ is the set of all possible mixed strategies of a trader:

$$
\Delta=\left\{\left\langle x_{1}, \ldots, x_{M}\right\rangle \in[0,1]^{M}: \sum_{m=1}^{M} x_{m} \leq 1\right\}
$$

Similarly, we use $\omega_{j}^{s}:[\underline{c}, \bar{c}] \times \mathcal{M} \rightarrow[0,1]$ to define the probability of selecting a marketplace of seller $j$, and write the complete strategy as:

$$
\delta_{j}^{s}\left(\theta^{s}\right)=\left\langle\omega_{j}^{s}\left(\theta^{s}, 1\right), \omega_{j}^{s}\left(\theta^{s}, 2\right), \ldots \omega_{j}^{s}\left(\theta^{s}, M\right)\right\rangle, \quad \delta_{j}^{s}\left(\theta^{s}\right) \in \Delta
$$

Now we use $\delta^{b}=\left\langle\delta_{1}^{b}(\cdot), \delta_{2}^{b}(\cdot), \ldots \delta_{B}^{b}(\cdot)\right\rangle$ to denote the strategy profile of buyers, and $\delta^{s}=\left\langle\delta_{1}^{s}(\cdot), \delta_{2}^{s}(\cdot), \ldots \delta_{S}^{s}(\cdot)\right\rangle$ that of the sellers. Given a buyers' strategy profile $\delta^{b}$ and a sellers' strategy profile $\delta^{s}$, the expected utility of a buyer $i$ with type $\theta^{b}$ is defined by:

$$
\tilde{U}_{i}^{b}\left(\mathcal{P}, \mathcal{K}, \delta^{b}, \delta^{s}, \theta^{b}\right)=\sum_{m=1}^{M} \omega_{i}^{b}\left(\theta^{b}, m\right) \times \tilde{U}_{i, m}^{b}\left(\mathcal{P}, \mathcal{K}, \delta^{b}, \delta^{s}, \theta^{b}\right)
$$

where $\tilde{U}_{i, m}^{b}\left(\mathcal{P}, \mathcal{K}, \delta^{b}, \delta^{s}, \theta^{b}\right)$ is buyer $i$ 's expected utility if it chooses to trade in the marketplace $m$, which depends on the specific matching policy adopted by marketplace $m$. The expected utility of the sellers is defined analogously. 


\section{Marketplace's Expected Profit}

In the above we have specified a general framework for analysing competing double auction marketplaces. Before we can analyse the equilibrium charging behaviour of marketplaces, we need to know marketplaces' expected profits given their fees and given the behaviour of the traders. In this section, we will describe how to calculate marketplaces' expected profits.

In order to calculate the marketplaces' expected profits, we need to know which bidding strategy traders will use to submit their offers and which matching policy marketplaces will use to match buyers and sellers to make transactions. Specifically, traders' bidding strategy and marketplaces' matching policy used in this work are specified as follows. As we did in [1], we assume that traders use a truthtelling bidding strategy, which means they will submit their types as their offers during the trading process. For the matching policy, we consider equilibrium matching since this aims to maximise traders' profits and thus maximises the allocative efficiency for the marketplace. In detail, this policy will match the buyer with $v$-th highest limit price with the seller with $v$-th lowest cost price if the seller's cost price is not greater than the buyer's limit price. Furthermore, we assume that traders with the same type will employ the same market selection strategy. Thus in the following, we omit the trader's index $i, j$ when it is intuitively clear.

Now in order to get insight from this complicated game with more traders and more types, we use the same simplifying assumptions made in [1]. We only consider the competition between two marketplaces, i.e. $M=2$. In order to allow for tractable results, we restrict our analysis to discrete trader types. In particular, we assume that there are two types of buyers and two types of sellers: rich and poor, which are denoted by $t_{2}^{b}$ and $t_{1}^{b}$ respectively for buyers, and $t_{1}^{s}$ and $t_{2}^{s}$ for sellers. A rich buyer is defined as having a higher limit price than a poor buyer, i.e. $t_{2}^{b}>t_{1}^{b}$, and a rich seller is defined as having a lower cost price than a poor seller, i.e. $t_{1}^{s}<t_{2}^{s}$. Trader types are independently drawn from the discrete uniform distribution (i.e. both types are equally likely). In addition, since we focus on how to set marketplace fees, we keep the pricing parameter $k_{m}=0.5$ $(m=1,2)$. Then marketplaces can only affect traders' market selections by changing fees.

Now we are ready to derive the equations to calculate marketplaces' expected profits given the fee system $\mathcal{P}$. Firstly, we calculate marketplaces' expected profit given traders' market selection strategies: $\omega^{b}\left(t_{1}^{b}, m\right), \omega^{b}\left(t_{2}^{b}, m\right), \omega^{s}\left(t_{1}^{s}, m\right)$ and $\omega^{s}\left(t_{2}^{s}, m\right)$. In order to do this, we calculate the probability that there are exactly $\tau_{1}^{b}$ poor buyers and $\tau_{2}^{b}$ rich buyers choosing the marketplace $m$ :

$\varrho_{m}^{b}\left(\tau_{1}^{b}, \tau_{2}^{b}\right)=\left(\begin{array}{c}B \\ \tau_{1}^{b}, \tau_{2}^{b}, B-\tau_{1}^{b}-\tau_{2}^{b}\end{array}\right) *\left(\frac{\omega^{b}\left(t_{1}^{b}, m\right)}{2}\right)^{\tau_{1}^{b}} *\left(\frac{\omega^{b}\left(t_{2}^{b}, m\right)}{2}\right)^{\tau_{2}^{b}} *\left(1-\frac{\omega^{b}\left(t_{1}^{b}, m\right)}{2}-\frac{\omega^{b}\left(t_{2}^{b}, m\right)}{2}\right)^{\left(B-\tau_{1}^{b}-\tau_{2}^{b}\right)}$

where $\left(\begin{array}{c}B \\ \tau_{1}^{b}, \tau_{2}^{b}, B-\tau_{1}^{b}-\tau_{2}^{b}\end{array}\right)$ is the multinomial coefficient, $\frac{\omega^{b}\left(t_{1}^{b}, m\right)}{2}$ is the probability that a buyer is poor and chooses marketplace $m$. Similarly, we get the probability 
that there are exactly $\tau_{1}^{s}$ rich sellers and $\tau_{2}^{s}$ poor sellers in the marketplace $m$ :

$\varrho_{m}^{s}\left(\tau_{1}^{s}, \tau_{2}^{s}\right)=\left(\begin{array}{c}S \\ \tau_{1}^{s}, \tau_{2}^{s}, S-\tau_{1}^{s}-\tau_{2}^{s}\end{array}\right) *\left(\frac{\omega^{s}\left(t_{1}^{s}, m\right)}{2}\right)^{\tau_{1}^{s}} *\left(\frac{\omega^{s}\left(t_{2}^{s}, m\right)}{2}\right)^{\tau_{2}^{s}} *\left(1-\frac{\omega^{s}\left(t_{1}^{s}, m\right)}{2}-\frac{\omega^{s}\left(t_{2}^{s}, m\right)}{2}\right)^{\left(S-\tau_{1}^{s}-\tau_{2}^{s}\right)}$

Furthermore, marketplace $m$ 's expected profit when there are exactly $\tau_{1}^{b}$ poor buyers, $\tau_{2}^{b}$ rich buyers, $\tau_{1}^{s}$ rich sellers and $\tau_{2}^{s}$ poor sellers in this marketplace is calculated by:

$$
\tilde{U}_{m}\left(\mathcal{P}, \tau_{1}^{b}, \tau_{2}^{b}, \tau_{1}^{s}, \tau_{2}^{s}\right)=\left(\tau_{1}^{b}+\tau_{2}^{b}\right) * p_{m}^{b}+\left(\tau_{1}^{s}+\tau_{2}^{s}\right) * p_{m}^{s}+\Lambda^{b} * q_{m}^{b}+\Lambda^{s} * q_{m}^{s}
$$

where $\Lambda^{b}, \Lambda^{s}$ are the buyers and sellers' share of the trading surplus respectively when $\tau_{1}^{b}$ poor buyers, $\tau_{2}^{b}$ rich buyers, $\tau_{1}^{s}$ rich sellers and $\tau_{2}^{s}$ poor sellers are matched according to the equilibrium matching policy ${ }^{3}$. At this moment, we can get the marketplace's expected profit given the traders' market selection strategies: $\omega^{b}\left(t_{1}^{b}, m\right), \omega^{b}\left(t_{2}^{b}, m\right), \omega^{s}\left(t_{1}^{s}, m\right)$ and $\omega^{s}\left(t_{2}^{s}, m\right)$ :

$$
\begin{aligned}
& \tilde{U}_{m}\left(\mathcal{P}, \omega^{b}\left(t_{1}^{b}, m\right), \omega^{b}\left(t_{2}^{b}, m\right), \omega^{s}\left(t_{1}^{s}, m\right), \omega^{s}\left(t_{2}^{s}, m\right)\right) \\
& =\sum_{\tau_{1}^{b}=0}^{B} \sum_{\tau_{2}^{b}=0}^{B-\tau_{1}^{b}} \sum_{\tau_{1}^{s}=0}^{S} \sum_{\tau_{2}^{s}=0}^{S-\tau_{1}^{s}} \varrho_{m}^{b}\left(\tau_{1}^{b}, \tau_{2}^{b}\right) * \varrho_{m}^{s}\left(\tau_{1}^{s}, \tau_{2}^{s}\right) * \tilde{U}_{m}\left(\mathcal{P}, \tau_{1}^{b}, \tau_{2}^{b}, \tau_{1}^{s}, \tau_{2}^{s}\right)
\end{aligned}
$$

Now given a fee system $\mathcal{P}$, we calculate the marketplaces' expected profits at the point where all traders use equilibrium market selection strategies. As we discussed in [1], there can exist multiple Nash equilibria of market selection strategies. In such cases, the marketplace's expected profit depends on which NEQ strategies traders will choose and the probability of choosing such NEQ strategies. In [1], given a fee system, we have used EGT to analyse how traders choose NEQ strategies. Similarly, in this paper, we use EGT to find which NEQ strategies traders will choose and with what probability.

In more detail, in EGT, players gradually adjust their strategies over time in response to the repeated observations of their opponents' strategies. In particular, the replicator dynamics equation is often used to specify the dynamic adjustment of the probability of which pure strategy should be played. Then in our work, the 4-population replicator equations (rich buyers, poor buyers, rich sellers and poor sellers) showing the dynamic changes of traders' selection strategies with respect to time $t$ are given by:

$$
\begin{aligned}
& \dot{\omega}^{b}\left(t_{1}^{b}, 1\right)=\frac{d \omega^{b}\left(t_{1}^{b}, 1\right)}{d t}=\left(\tilde{U}_{1}^{b}\left(\mathcal{P}, \mathcal{K}, \delta^{b}, \delta^{s}, t_{1}^{b}\right)-\tilde{U}^{b}\left(\mathcal{P}, \mathcal{K}, \delta^{b}, \delta^{s}, t_{1}^{b}\right)\right) * \omega^{b}\left(t_{1}^{b}, 1\right) \\
& \dot{\omega}^{b}\left(t_{2}^{b}, 1\right)=\frac{d \omega^{b}\left(t_{2}^{b}, 1\right)}{d t}=\left(\tilde{U}_{1}^{b}\left(\mathcal{P}, \mathcal{K}, \delta^{b}, \delta^{s}, t_{2}^{b}\right)-\tilde{U}^{b}\left(\mathcal{P}, \mathcal{K}, \delta^{b}, \delta^{s}, t_{2}^{b}\right)\right) * \omega^{b}\left(t_{2}^{b}, 1\right) \\
& \dot{\omega}^{s}\left(t_{1}^{s}, 1\right)=\frac{d \omega^{s}\left(t_{1}^{s}, 1\right)}{d t}=\left(\tilde{U}_{1}^{s}\left(\mathcal{P}, \mathcal{K}, \delta^{b}, \delta^{s}, t_{1}^{s}\right)-\tilde{U}^{s}\left(\mathcal{P}, \mathcal{K}, \delta^{b}, \delta^{s}, t_{1}^{s}\right)\right) * \omega^{s}\left(t_{1}^{s}, 1\right) \\
& \dot{\omega}^{s}\left(t_{2}^{s}, 1\right)=\frac{d \omega^{s}\left(t_{2}^{s}, 1\right)}{d t}=\left(\tilde{U}_{1}^{s}\left(\mathcal{P}, \mathcal{K}, \delta^{b}, \delta^{s}, t_{2}^{s}\right)-\tilde{U}^{s}\left(\mathcal{P}, \mathcal{K}, \delta^{b}, \delta^{s}, t_{2}^{s}\right)\right) * \omega^{s}\left(t_{2}^{s}, 1\right)
\end{aligned}
$$

\footnotetext{
3 This can be easily calculated. For example, when there are 2 rich buyers, 3 poor buyers, 3 rich sellers and 2 poor buyers in the marketplace $m, \Lambda^{b}=\left(\max \left(t_{2}^{b}-t_{1}^{s}, 0\right) * 2+\max \left(t_{1}^{b}-t_{1}^{s}, 0\right)+\right.$ $\left.\max \left(t_{1}^{b}-t_{2}^{s}, 0\right) * 2\right) * k_{m}$ and $\Lambda^{s}=\left(\max \left(t_{2}^{b}-t_{1}^{s}, 0\right) * 2+\max \left(t_{1}^{b}-t_{1}^{s}, 0\right)+\max \left(t_{1}^{b}-t_{2}^{s}, 0\right) * 2\right) *\left(1-k_{m}\right)$.
} 
As an example, $\dot{\omega}^{b}\left(t_{1}^{b}, 1\right)$ describes how the poor buyer with type $t_{1}^{b}$ changes its probability of choosing marketplace 1 . Here, $\tilde{U}_{1}^{b}\left(\mathcal{P}, \mathcal{K}, \delta^{b}, \delta^{s}, t_{1}^{b}\right)$ is the poor buyer's expected utility when choosing marketplace 1 given other traders' strategies, and $\tilde{U}^{b}\left(\mathcal{P}, \mathcal{K}, \delta^{b}, \delta^{s}, t_{1}^{b}\right)$ is the poor buyer's overall expected utility. Given that traders use the truthtelling bidding strategy and marketplaces use the equilibrium matching policy, these equations have been derived in [1]. In order to get the dynamics of the strategies, we need to calculate trajectories, which indicate how the mixed strategies evolve. In more detail, initially, a mixed strategy is chosen as a starting point. For convenience, we use $\left(\omega^{b}\left(t_{2}^{b}, 1\right), \omega^{b}\left(t_{1}^{b}, 1\right), \omega^{s}\left(t_{1}^{s}, 1\right), \omega^{s}\left(t_{2}^{s}, 1\right)\right)$ to represent this starting point. The dynamics are then calculated according to the above replicator equations. According to the dynamic changes of traders' strategies, their current mixed strategy can be calculated. Such calculations are repeated until $\dot{\omega}^{b}(\cdot, 1)$ and $\dot{\omega}^{s}(\cdot, 1)$ become zero, at which point the equilibrium is reached. The replicator dynamics show the trajectories and how they converge to an equilibrium. When considering traders evolving from all possible starting points, we get several regions. The region where all trajectories converge to a particular equilibrium is called the basin of attraction of this equilibrium. The basin is very useful since its size indicates the probability of traders converging to that equilibrium, which is necessary for calculating marketplaces' expected profits.

However, since probabilities are continuous from 0 to 1 , there are infinitely many mixed strategies available to each type of trader. Thus the set of possible starting points is also infinite, which results in the difficulty of accurately calculating the sizes of basins. In this work, we have to discretize the starting points to approximate the size of basin of attraction to each NEQ. By so doing, we know the probability of traders converging to each equilibrium. For example, in this paper we calculate the size of basin of attraction by discretizing the mixed strategy of each type from 0.01 to 0.99 with step size 0.049 , which gives $21^{4}=194481$ different starting points. Note that if we use even more points, we can estimate the probability of traders' convergence to each equilibrium more accurately.

Now that we know, given a fee system $\mathcal{P}$, what NEQ strategies traders will choose and with what probabilities. We calculate the expected profit for a marketplace. Specifically, given that there are $X$ possible NEQs market selection strategies, we use $\left\langle x_{1}, x_{2}, \ldots, x_{X}\right\rangle$ to represent the probabilities of traders converging to these NEQs. Then the marketplace $m$ 's expected profit in the fee system $\mathcal{P}$ is:

$$
\tilde{U}_{m}(\mathcal{P})=\sum_{z=1}^{X} x_{z} * \tilde{U}_{m}\left(\mathcal{P}, \omega^{z b}\left(t_{1}^{b}, m\right), \omega^{z b}\left(t_{2}^{b}, m\right), \omega^{z s}\left(t_{1}^{s}, m\right), \omega^{z s}\left(t_{2}^{s}, m\right)\right)
$$

where $\omega^{z b}\left(t_{1}^{b}, m\right), \omega^{z b}\left(t_{2}^{b}, m\right), \omega^{z s}\left(t_{1}^{s}, m\right)$ and $\omega^{z s}\left(t_{2}^{s}, m\right)$ denote the $z$-th NEQ market selection strategies. 


\section{Equilibrium Analysis of Market Fees}

Given the equations to calculate the marketplaces' expected profits, we now define a Nash equilibrium for the marketplaces. Since the strategies of each marketplace consist of the range of possible fees, an equilibrium constitutes a fee system $\mathcal{P}$. Specifically, the Nash equilibrium fee system in our setting is defined as follows:

Definition 1 The fees system $\mathcal{P}^{*}=\left\langle\mathcal{P}_{m}^{*}, \mathcal{P}_{-m}^{*}\right\rangle$ constitutes a Nash equilibrium fee system, if $\forall m \in \mathcal{M}, \forall \mathcal{P}_{m} \in \Psi$,

$$
\begin{gathered}
\tilde{U}_{m}\left(\mathcal{P}^{*}\right)=\sum_{z=1}^{X^{*}} x_{z}^{*} * \tilde{U}_{m}\left(\left\langle\mathcal{P}_{m}^{*}, \mathcal{P}_{-m}^{*}\right\rangle, \omega^{z b *}\left(t_{1}^{b}, m\right), \omega^{z b *}\left(t_{2}^{b}, m\right), \omega^{z s *}\left(t_{1}^{s}, m\right), \omega^{z s *}\left(t_{2}^{s}, m\right)\right) \\
\geq \tilde{U}_{m}(\mathcal{P})=\sum_{z=1}^{X} x_{z} * \tilde{U}_{m}\left(\left\langle\mathcal{P}_{m}, \mathcal{P}_{-m}^{*}\right\rangle, \omega^{z b}\left(t_{1}^{b}, m\right), \omega^{z b}\left(t_{2}^{b}, m\right), \omega^{z s}\left(t_{1}^{s}, m\right), \omega^{z s}\left(t_{2}^{s}, m\right)\right)
\end{gathered}
$$

where $\Psi$ is the set of all possible fees.

We now analyse this equilibrium in detail. First, we need to calculate the marketplaces' expected profits given different fee systems. As we know, the range of possible fees is continuous, which results in infinitely many possible fee systems. In [7], researchers claim that for this kind of game, it is useful to approximate the game by restricting the strategy space, and results from the restricted strategy space still provide insights into the original game. Similarly in this paper, in order to obtain tractable results, we also restrict the fee space by discretizing these fees. For example, we discretize profit and registration fees from 0 to 1 with step size 0.1. Then we can calculate marketplaces' expected profits corresponding to these fees, and generate an expected profit matrix for marketplaces, by which we can analyse the equilibrium fee system.

We now analyse this issues in two cases. Specifically, in the first case, we consider two competing marketplaces that only charge the profit fees. In the second case, we consider a setting where one marketplace charges the registration fee, and the other marketplace charges the profit fee.

\subsection{Both Marketplaces Charging Profit Fees}

First we consider the case that only profit fees can be charged to traders. We assume that there are 5 buyers and 5 sellers, and the surpluses of buyers and sellers are symmetric. Specifically, we let $t_{1}^{b}=4, t_{2}^{b}=6, t_{1}^{s}=0$ and $t_{2}^{s}=2$. Furthermore, we assume that competing marketplaces charge the same profit fee to buyers and sellers (i.e. $\left.q_{m}^{b}=q_{m}^{s}\right)^{4}$. Now we discretize profit fee from 0 to 1

\footnotetext{
4 At this moment, rich(poor) buyer and rich(poor) seller have the same behaviour of selecting marketplaces. Then Equations 6 and 9 are consistent and Equations 7 and 8 are consistent. By so doing, we reduce 4-population replicator dynamics to 2 -population replicator dynamics. This is convenient for visualising how traders evolve their strategies and approximating the size of basins of attraction in a 2-dimensional space.
} 


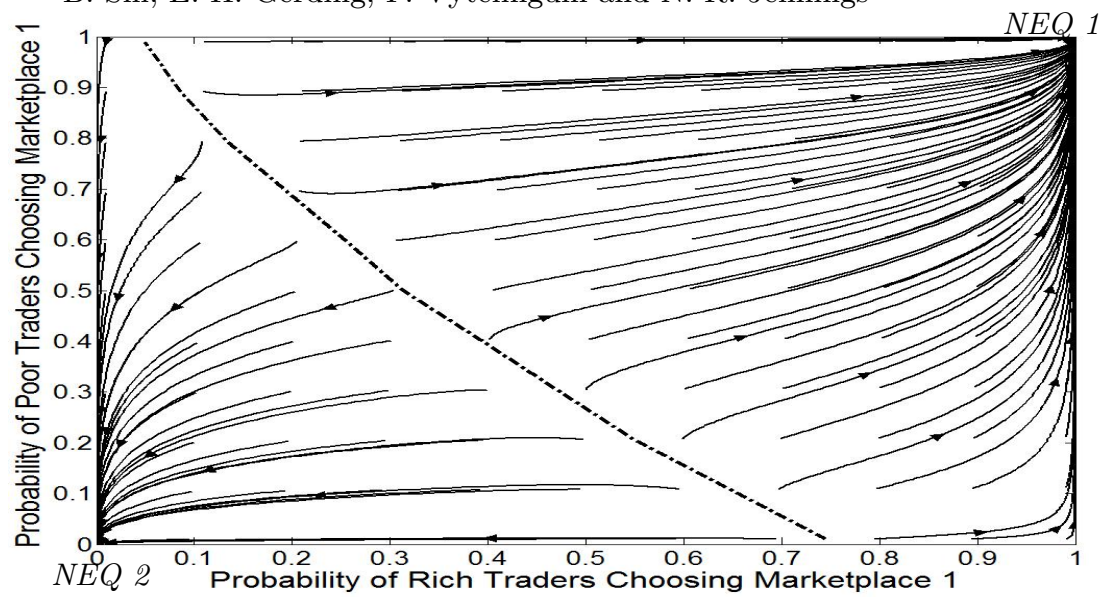

Fig. 1. Evolutionary process when both marketplaces 1 and 2 charge profit fees. The dotted line denotes the boundary between the basins of attractions.

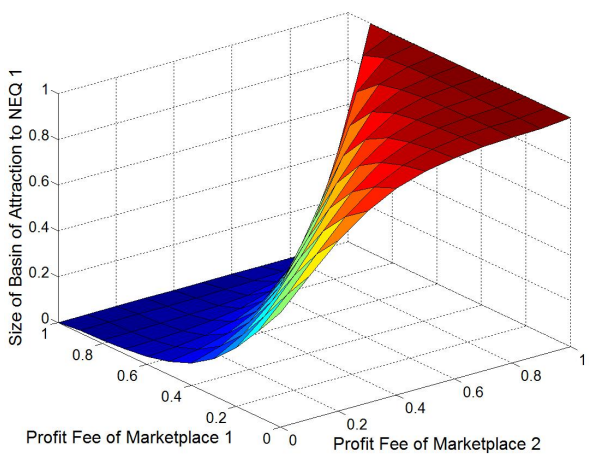

(a) Size of basin of attraction to NEQ 1.

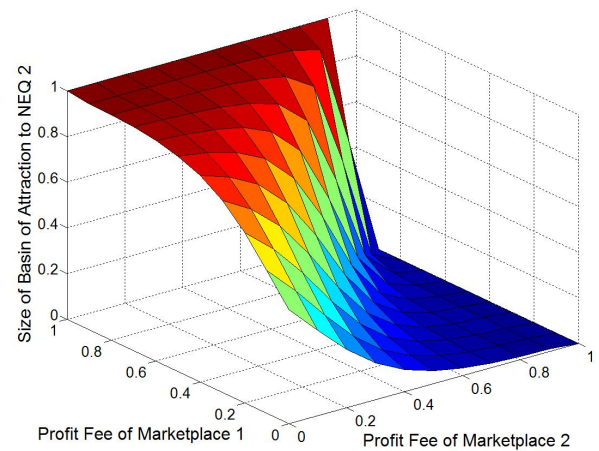

(b) Size of basin of attraction to NEQ 2.

Fig. 2. Sizes of basins of attraction with respect to different fees.

with step size 0.1 . Therefore each marketplace can choose from 11 different profit fees. For two competing marketplaces, there are $11^{2}=121$ different fee systems. For each of these combinations, we use EGT to obtain the basin of attraction to each NEQ of market selection strategies. Then by approximating the size of each basin, we get the probability of traders choosing each NEQ. For example, when marketplace 1 charges $20 \%$ profit fee and marketplace 2 charges $30 \%$ profit fee, the basins of attraction are shown in Fig. $1^{5}$. From this figure, we find that all traders will converge to marketplace 1 (NEQ 1) or marketplace 2 (NEQ 2). By approximating the size of each basin of attraction, we can determine the probability of traders converging to each NEQ (the probabilities to NEQ 1 and NEQ 2 are 0.658 and 0.342 respectively in this case).

Now we explore traders' evolutionary process in all fee systems. The probability of traders choosing each NEQ of market selection strategies corresponding to all fee systems are shown in Fig. 2. Then using Equation 10, we calculate the marketplaces' expected profits. The results are shown in Table 1. From this table,

\footnotetext{
5 Here we discretize the mixed strategy of each type of trader from 0.01 to 0.99 with a bigger step size, 0.098 , for clearly visualising purpose.
} 


\begin{tabular}{|c|c|c|c|c|c|c|c|c|c|c|c|}
\hline & $\mathbf{0 . 0}$ & $\mathbf{0 . 1}$ & $\mathbf{0 . 2}$ & $\mathbf{0 . 3}$ & $\mathbf{0 . 4}$ & $\mathbf{0 . 5}$ & $\mathbf{0 . 6}$ & $\mathbf{0 . 7}$ & $\mathbf{0 . 8}$ & $\mathbf{0 . 9}$ & $\mathbf{1 . 0}$ \\
\hline $\mathbf{0 . 0}$ & $0.00,0.00$ & $0.00,0.75$ & $0.00,1.02$ & $0.00,0.97$ & $0.00,0.78$ & $0.00,0.58$ & $0.00,0.42$ & $0.00,0.29$ & $0.00,0.22$ & $0.00,0.20$ & $0.00,0.00$ \\
\hline $\mathbf{0 . 1}$ & $0.75,0.00$ & $1.00,1.00$ & $1.28,1.44$ & $1.54,1.39$ & $1.73,1.10$ & $1.85,0.77$ & $1.91,0.52$ & $1.95,0.34$ & $1.97,0.24$ & $1.98,0.20$ & $2.00,0.00$ \\
\hline $\mathbf{0 . 2}$ & $1.02,0.00$ & $1.44,1.28$ & $2.00,2.00$ & $2.63,2.05$ & $3.18,1.64$ & $3.56,1.11$ & $3.77,0.70$ & $3.88,0.43$ & $3.93,0.27$ & $3.95,0.22$ & $4.00,0.00$ \\
\hline $\mathbf{0 . 3}$ & $0.97,0.00$ & $1.39,1.54$ & $2.05,2.63$ & $\mathbf{3 . 0 0 , 3 . 0 0}$ & $4.07,2.58$ & $4.97,1.72$ & $5.50,1.01$ & $5.76,0.56$ & $5.88,0.32$ & $5.93,0.22$ & $6.00,0.00$ \\
\hline $\mathbf{0 . 4}$ & $0.78,0.00$ & $1.10,1.73$ & $1.64,3.18$ & $2.58,4.07$ & $4.00,4.00$ & $5.66,2.93$ & $6.90,1.64$ & $7.54,0.81$ & $7.81,0.38$ & $7.90,0.22$ & $8.00,0.00$ \\
\hline $\mathbf{0 . 5}$ & $0.58,0.00$ & $0.77,1.85$ & $1.11,3.56$ & $1.72,4.97$ & $2.93,5.66$ & $5.00,5.00$ & $7.45,3.06$ & $9.03,1.36$ & $9.65,0.56$ & $9.86,0.25$ & $10.00,0.00$ \\
\hline $\mathbf{0 . 6}$ & $0.42,0.00$ & $0.52,1.91$ & $0.70,3.77$ & $1.01,5.50$ & $1.64,6.90$ & $3.06,7.45$ & $6.00,6.00$ & $9.54,2.87$ & $11.30,0.93$ & $11.80,0.31$ & $12.00,0.00$ \\
\hline $\mathbf{0 . 7}$ & $0.29,0.00$ & $0.34,1.95$ & $0.43,3.88$ & $0.56,5.76$ & $0.81,7.54$ & $1.36,9.03$ & $2.87,9.54$ & $7.00,7.00$ & $12.08,2.19$ & $13.66,0.43$ & $14.00,0.00$ \\
\hline $\mathbf{0 . 8}$ & $0.22,0.00$ & $0.24,1.97$ & $0.27,3.93$ & $0.32,5.88$ & $0.38,7.81$ & $0.56,9.65$ & $0.93,11.30$ & $2.19,12.08$ & $8.00,8.00$ & $15.07,1.04$ & $16.00,0.00$ \\
\hline $\mathbf{0 . 9}$ & $0.20,0.00$ & $0.20,1.98$ & $0.22,3.95$ & $0.22,5.93$ & $0.22,7.90$ & $0.25,9.86$ & $0.31,11.80$ & $0.43,13.66$ & $1.04,15.07$ & $9.00,9.00$ & $18.00,0.00$ \\
\hline $\mathbf{1 . 0}$ & $0.00,0.00$ & $0.00,2.00$ & $0.00,4.00$ & $0.00,6.00$ & $0.00,8.00$ & $0.00,10.0$ & $0.00,12.00$ & $0.00,14.00$ & $0.00,16.00$ & $0.00,18.00$ & $10.00,10.00$ \\
\hline
\end{tabular}

Table 1. Profits of marketplace 1 and marketplace 2. The first column is the profit fee of marketplace 1 and the first row is the profit fee of marketplace 2 . The first element in each cell is marketplace 1's expected profit, and the second is marketplace 2's expected profit. Bold italic fees constitute a NEQ fee system.

by using Gambit ${ }^{6}$, we find that both marketplaces charging $30 \%$ profit fee constitutes a unique pure NEQ fee system. Interestingly, in this equilibrium, both competing marketplaces charge non-zero profit fees and therefore make positive profits. This contrasts with competition between one-sided marketplaces (such as the classical Bertrand competition), in which competing marketplaces reduce their fees to their cost level and make zero profit in equilibrium [9]. This is because, in two-sided double auction marketplaces, there is still a probability that the traders converge to the marketplace which charges slightly higher fees compared to its opponent (as can be seen in Fig. 2).

Finally, we also analyse the case when both competing marketplaces charge registration fees. In this case, the traders' expected profits on both marketplaces may be negative, and then traders will not enter any marketplaces. However, other conclusions are similar to the case that both competing marketplaces charge profit fees. In particularly, we still find traders eventually converge to one marketplace. The same result holds if we change traders' types and the number of traders.

\subsection{Asymmetric Market Fees}

In this section, we will consider the case that different competing marketplaces charge different types of fees: marketplace 1 charges the profit fee, and marketplace 2 charges the registration fee. In previous section, we find that traders always converge to one of two equilibria when they are limited to charging the same type of fees. This means that marketplaces cannot co-exist when traders are in equilibrium. In this section, we first analyse whether competing marketplaces can co-exist when different types of fees are allowed. Then we analyse the equilibrium fee system.

We still assume that competing marketplaces charge the same fee to buyers and sellers (i.e. $p_{m}^{b}=p_{m}^{s}$ and $q_{m}^{b}=q_{m}^{s}$ ). Then we discretize registration and profit fees from 0 to 1 with step size 0.1 . Then there are again 121 different fee systems. By evolving traders' strategies from different starting points and under different fee systems, we find co-existence of competing marketplaces. For example, when marketplace 1 charges $50 \%$ profit fee and marketplace 2

\footnotetext{
${ }^{6}$ http://gambit. sourceforge.net/
} 


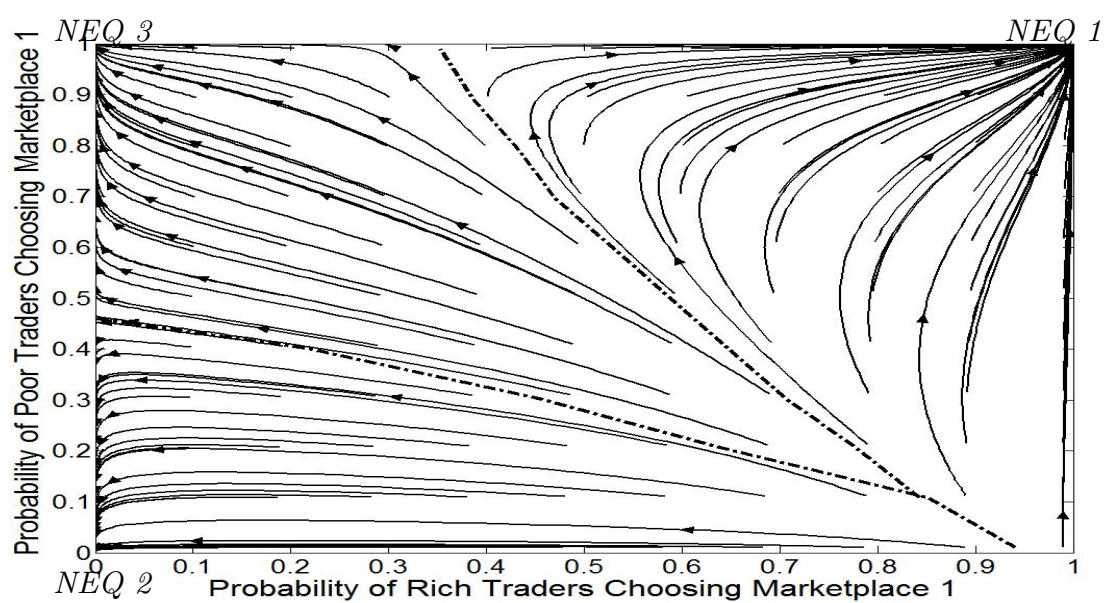

Fig. 3. Evolutionary process when marketplace 1 charges profit fee and marketplace 2 charges registration fee. The dotted line denotes the boundarv between the basins of attractions.

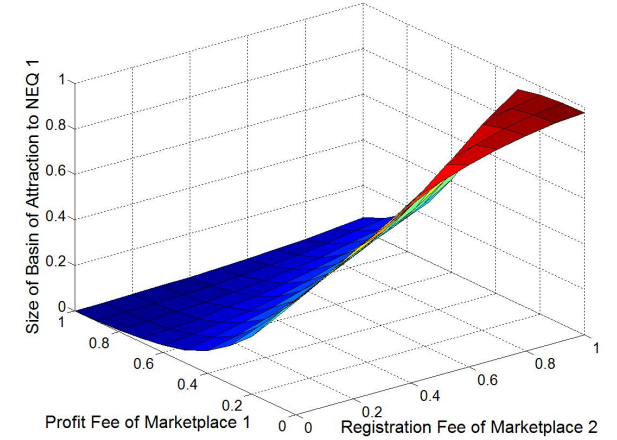

(a) Size of basin of attraction to NEQ 1.

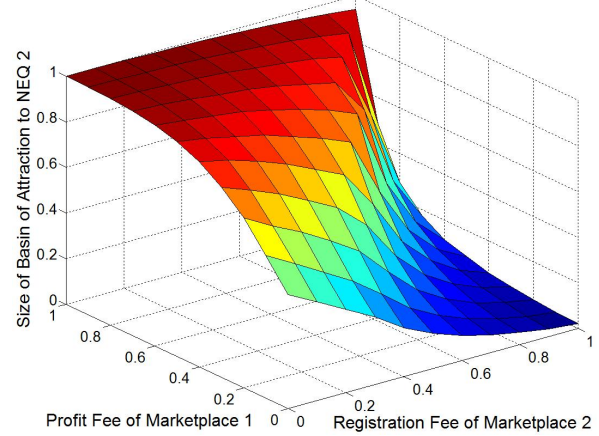

(b) Size of basin of attraction to NEQ 2 .

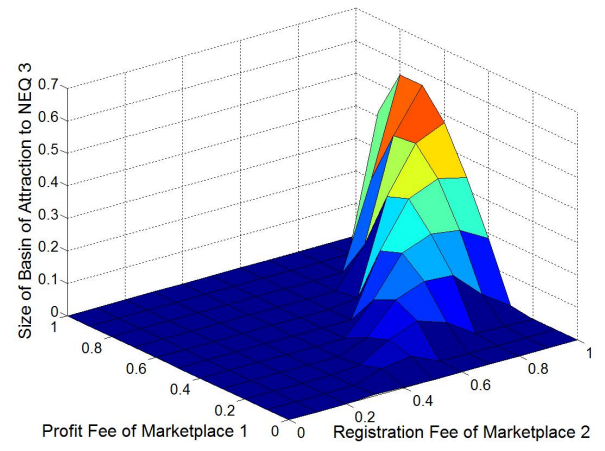

(c) Size of basin of attraction to NEQ 3.

Fig. 4. Sizes of basins of attraction with respect to different fees.

charges 0.8 registration fee, the evolution of traders' selection strategies from different starting points is shown in Fig. 3. From this figure, we find that there are now three basins of attraction: all traders converge to marketplace 1 (NEQ 1 ); all traders converge to marketplace 2 (NEQ 2); rich traders converge to marketplace 2, and poor traders converge to marketplace 1 (NEQ 3), i.e. two competing marketplaces co-exist in equilibrium. By exploring traders' market 


\begin{tabular}{|l|c|c|c|c|c|c|c|c|c|c|c|}
\hline & $\mathbf{0 . 0}$ & $\mathbf{0 . 1}$ & $\mathbf{0 . 2}$ & $\mathbf{0 . 3}$ & $\mathbf{0 . 4}$ & $\mathbf{0 . 5}$ & $\mathbf{0 . 6}$ & $\mathbf{0 . 7}$ & $\mathbf{0 . 8}$ & $\mathbf{0 . 9}$ & $\mathbf{1 . 0}$ \\
\hline $\mathbf{0 . 0}$ & $0.00,0.00$ & $0.00,0.43$ & $0.00,0.73$ & $0.00,0.90$ & $0.00,0.86$ & $0.00,0.77$ & $0.00,0.67$ & $0.00,0.54$ & $0.00,0.43$ & $0.00,0.31$ & $0.00,0.23$ \\
\hline $\mathbf{0 . 1}$ & $0.75,0.00$ & $0.89,0.56$ & $1.03,0.97$ & $1.18,1.22$ & $1.32,1.32$ & $1.49,1.20$ & $1.69,0.92$ & $1.79,0.73$ & $1.86,0.54$ & $1.91,0.39$ & $1.95,0.27$ \\
\hline $\mathbf{0 . 2}$ & $1.02,0.00$ & $1.31,0.67$ & $1.55,1.22$ & $1.84,1.62$ & $2.15,1.85$ & $2.48,1.81$ & $2.83,1.59$ & $3.22,1.22$ & $3.62,0.75$ & $3.76,0.54$ & $3.86,0.36$ \\
\hline $\boldsymbol{0 . 3}$ & $1.00,0.00$ & $1.29,0.79$ & $1.59,1.47$ & $1.97,2.01$ & $2.37,2.42$ & $\mathbf{2 . 8 8 , 2 . 5 5}$ & $3.44,2.33$ & $4.00,1.99$ & $4.49,1.64$ & $5.20,1.01$ & $5.67,0.53$ \\
\hline $\mathbf{0 . 4}$ & $0.80,0.00$ & $1.03,0.87$ & $1.34,1.66$ & $1.72,2.36$ & $2.19,2.90$ & $2.66,3.34$ & $3.35,3.30$ & $4.17,2.89$ & $4.91,2.48$ & $5.62,2.03$ & $6.32,1.53$ \\
\hline $\mathbf{0 . 5}$ & $0.57,0.00$ & $0.79,0.92$ & $1.07,1.79$ & $1.36,2.59$ & $1.77,3.29$ & $2.20,3.90$ & $2.72,4.37$ & $3.71,3.99$ & $4.62,3.49$ & $5.56,2.99$ & $6.38,2.56$ \\
\hline $\mathbf{0 . 6}$ & $0.38,0.00$ & $0.54,0.96$ & $0.76,1.87$ & $0.92,2.77$ & $1.28,3.57$ & $1.66,4.31$ & $2.12,4.94$ & $2.64,5.44$ & $3.92,4.64$ & $4.99,4.03$ & $5.97,3.54$ \\
\hline $\mathbf{0 . 7}$ & $0.22,0.00$ & $0.32,0.98$ & $0.48,1.93$ & $0.70,2.85$ & $0.85,3.76$ & $1.15,4.59$ & $1.53,5.35$ & $1.93,6.03$ & $2.73,6.14$ & $4.09,5.18$ & $5.19,4.55$ \\
\hline $\mathbf{0 . 8}$ & $0.11,0.00$ & $0.18,0.99$ & $0.29,1.96$ & $0.37,2.93$ & $0.54,3.86$ & $0.77,4.76$ & $1.01,5.62$ & $1.26,6.45$ & $1.71,7.14$ & $2.86,6.66$ & $4.35,5.54$ \\
\hline $\mathbf{0 . 9}$ & $0.04,0.00$ & $0.09,1.00$ & $0.16,1.98$ & $0.20,2.97$ & $0.29,3.94$ & $0.45,4.88$ & $0.61,5.80$ & $0.81,6.69$ & $1.10,7.51$ & $1.48,8.26$ & $3.24,6.92$ \\
\hline $\mathbf{1 . 0}$ & $0.00,0.00$ & $0.00,1.00$ & $0.04,2.00$ & $0.10,2.99$ & $0.14,3.97$ & $0.22,4.95$ & $0.36,5.89$ & $0.50,6.83$ & $0.68,7.73$ & $0.90,8.60$ & $1.18,9.41$ \\
\hline
\end{tabular}

Table 2. Profits of marketplace 1 and marketplace 2. The first column is the profit fee of marketplace 1 and the first row is the registration fee of marketplace 2. The first element in each cell is marketplace 1's expected profit, and the second is marketplace 2's expected profit. Bold italic fees constitute a NEQ fee system.

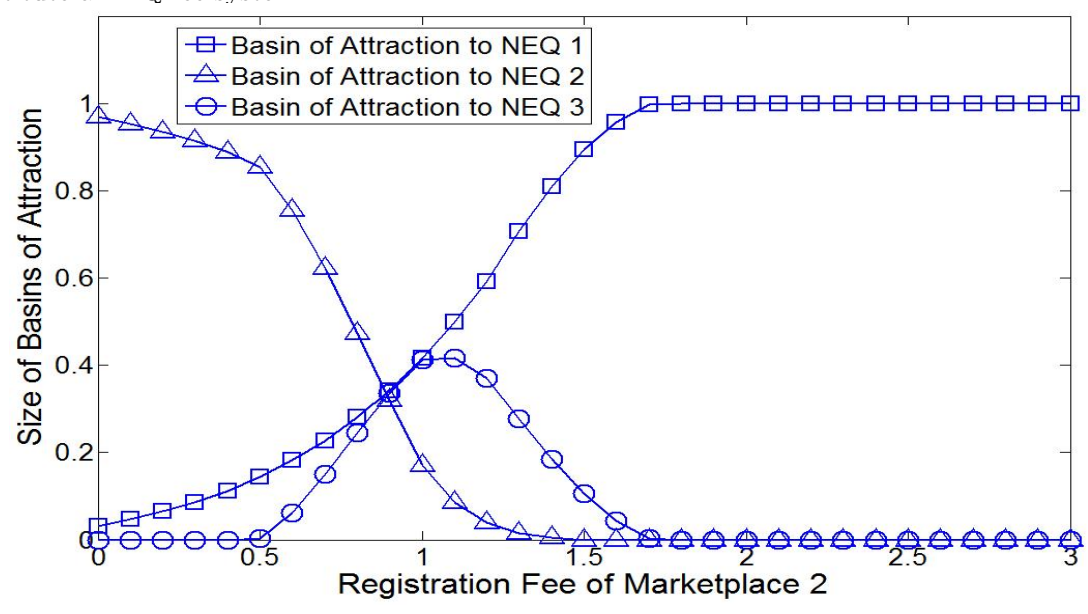

Fig. 5. Size of basins of attraction with respect to changed registration fees.

selection strategies under all possible fee systems, we obtain the probabilities of traders converging to each NEQ, which are shown in Fig. 4. Fig. 4(c) shows in which fee systems, traders may converge to different marketplaces.

After estimating the probabilities of traders' convergence to each NEQ, we then calculate the marketplaces' expected profits using Equation 10. The marketplaces' expected profits are shown in Table 2, from which we can see that in this case, marketplace 1 charging $30 \%$ profit fee, and marketplace 2 charging 0.5 registration fee constitutes the unique NEQ fee system.

Now we analyse how registration and profit fees affect the market selections of traders with different types. We note that, since the surplus of transaction between rich traders is higher than that between poor traders, then for the same profit fee, rich traders will lose more absolute profits than poor traders. Specifically, we let marketplace 1 charge $60 \%$ profit fee, marketplace 2 charge registration fee from 0 to 3 with step size 0.1 . The sizes of basins of attraction with respect to the registration fee are shown in Fig. 5. From the figure, we can see that when the registration fee is low, traders prefer to choose marketplace 2. This is shown by the line with triangle, which is above two other lines in the beginning. Then as the registration fee increases, poor traders will choose to leave marketplace 2 since increased registration fee cause decreased and even negative 
profits for them. Rich traders may still prefer marketplace 2 since compared to marketplace 1 which extracts more absolute profits by charging the profit fee, marketplace 2 is still cheaper. This is shown by the increased lines with circle and square when the registration fee increases from 0 to about 1 . However, when the registration fee becomes very high, both rich and poor traders will leave since their profits will be negative if they still choose to stay. This is shown by the decreased lines with triangle and circle and the increased line with square when the registration fee increases from 1 to 3 .

\section{Conclusions}

In this paper, we provided an approach to estimate marketplaces' expected profits for a given fee system, based on the equilibrium of the traders' market selection strategies, and the likelihood of various equilibria occurring. We then analysed the NEQ fee system in two different cases: where marketplaces charge the same type of fees and where they charge different types of fees. Such analysis is useful to guide the charging behaviour of competing marketplaces. For the settings analysed in this paper, we found that a pure Nash equilibrium always exists in which both competing marketplaces charge non-zero fees. In addition, we found that when one marketplace charges the registration fee and the other charges the profit fee, two competing marketplace may co-exist in which the traders are in equilibrium. In this equilibrium, rich traders converge to the marketplace charging the registration fee, and poor traders converge to the marketplace charging the profit fee.

In this analysis, we have assumed that traders use the truthtelling bidding strategy, which results in traders' true profits being revealed to the marketplaces. However, when traders adopt a bidding strategy that can shade their true types, traders can keep more profits even when the profit fee is high. In the future, we intend to analyse the equilibrium fee system in this case. Moreover, in practice, traders may never converge to any NEQ market selection strategies since competing marketplaces may keep adapting their fees. In this dynamic process, we want to address how competing marketplaces dynamically change their fees corresponding to the opponents' fees and traders' current market selections. Furthermore, we also would like to generalise our analysis of the traders' equilibrium behaviour of market selection strategies as well as the bidding strategies by considering traders with continuous types, and then analyse how to find a NEQ fee system for competing marketplaces.

\section{References}

1. Shi, B., Gerding, E. H., Vytelingum, P., Jennings, N. R.: A Game-Theoretic Analysis of Market Selection Strategies for Competing Double Auction Marketplaces. Proc. 9th Int Conf. on Autonomous Agents and Multi-Agent Systems (2010)

2. Lee, R. S.: Competing Platforms. http://pages.stern.nyu.edu/ rslee/papers. Department of Economics, NYU Stern School of Business and Yahoo! Research (2008)

3. Friedman, D., Rust, J.: The double auction market: institutions, theories and evidence. XIV of Santa Fe Institute Studies in the Science of Complexity. Perseus Publishing (1993)

4. Gerding, E. H., McBurney, P., Niu, J., Parsons, S., Phelps, S.: Overview of CAT: A market design competition. Technical Report, Dept. of Computer Science, University of Liverpool (2007)

5. Caillaud, B., Jullien, B.: Chicken \& egg: Competition among intermediation service providers. The RAND Journal of Economics. 34(2), 309-328 (2003) 
6. Rochet, J., Tirole, J.: Platform Competition in Two-Sided Markets. Journal of European Economic Association. 1(4), 990-1029 (2003)

7. Wellman, M. P.: Methods for empirical game-theoretic analysis. Proc. 21st National Conference on Artificial Intelligence. 1552-1555 (2006)

8. Armstrong, M.: Competition in Two-Sided Markets. The RAND Journal of Economics. 37(3), 668-691 (2006)

9. Bertrand, J.: Book review of theorie mathematique de la richesse sociale and of recherches sur les principles mathematiques de la theorie des richesses. Journal de Savants. 67, 499-508 (1883)

10. Weibull, J. W.: Evolutionary Game Theory. The MIT Press (1996) 